\title{
Location-aware and Superimposed-Pilot based Channel Estimation of Sparse HAP Radio Communication Channels
}

\author{
${ }^{\S}$ Syed Junaid Nawaz, ${ }^{\S}$ Babar Mansoor, ${ }^{\dagger}$ Shree K. Sharma, ${ }^{\S}$ Sardar M. Gulfam, and ${ }^{\ddagger}$ Mohmammad N. Patwary

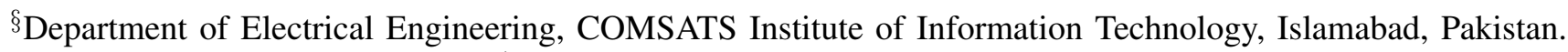 \\ †University of Western Ontario, Canada. \\ ${ }^{\ddagger}$ Faculty of Computing Engineering and Sciences, Staffordshire University, Stoke-on-trent, UK. \\ Email: junaidnawaz@ieee.org, sshar323@uwo.ca, babar_mansoor@comsats.edu.pk, \\ sardar_muhammad@comsats.edu.pk, and m.n.patwary@staffs.ac.uk.
}

\begin{abstract}
A superimposed (arithmetically added) Pilot (SiP) sequence based channel estimation method for beamforming assisted multi-antenna High Altitude Platform (HAP) land mobile radio communication systems is proposed, which exploits the prior available information of users' spatial location, density of users, and beam-width of HAP directional antenna. A thorough characterization of HAP sparse multipath radio propagation channels' is presented in first part of the paper, where mathematical relationship of HAP antenna beam-width with channel's delay span and optimal length of SiP base sequence are presented. Further, a location information aided and lowpower SiP sequence based Stage-wise Orthogonal Match Pursuit (StOMP) algorithm is proposed for estimation of channels from single-antenna user terminals to beamforming assisted large scale multiple-antenna HAP. A thorough analysis on the basis of Normalized Channel Mean Square Error (NCMSE) and Bit Error Rate (BER) performance of proposed method is presented; where the effect of channels' sparsity level, Pilot-to-Information power Ratio (PIR), beam-width of HAP's directional antenna, amount of HAP antenna elements, density of interfering users, and spatial location of active user terminal are thoroughly studied. A comparison of the proposed method with a notable reference technique available in the literature is also presented.
\end{abstract}

Keywords: HAP, sparse, superimposed pilot, compressed sensing, matching pursuit, channel estimation.

\section{INTRODUCTION}

One of the promising ways of improving latency, scalability and the robustness of current wireless networks is to utilize the highly precise knowledge of location information while designing and optimizing various system parameters of the Fifth Generation (5G) wireless networks. This knowledge can be obtained either with the help of Global Navigation Satellite Systems (GNSS) supported by different terrestrial systems and complemented by the existing Global Positioning System (GPS) or via various local radio-based localization technologies such as ultra-wide band, ZigBee, Bluetooth, WiFi, and Radio Frequency Identification (RFID) in the scenarios where GNSS signal is either unavailable or weak [1].

The design parameters of wireless communication systems are closely related to the location information in various forms such as delay, propagation distance, angle of arrival, user mobility, elevation angle, terrain structure, and predictable user behavior [1]. Therefore, location awareness is significantly helpful in various wireless networks such as Cognitive Radio (CR) networks, wireless sensor networks, cellular as well as ad-hoc networks [2]. For example, in CR scenarios, one of the main objectives is to acquire the precise knowledge about the spectrum usage in the surrounding environment and to exploit the available opportunities in an effective manner [3]. After obtaining the propagation characteristics of the surrounding environment with the help of location awareness capability (geolocation database or spectrum sensing), the CR node can adjust its transmission parameters in order to optimize its performance as well as to manage the harmful interference in the network [4]. Out of several benefits from the knowledge of location information in various application scenarios, this paper focuses on the application of location aware channel estimation in High Altitude Platform (HAP) based radio communication channels.

Recently, there has been an increasing attention to provide broadband services over wide geographical region with the help of HAPs. For example, the Google Balloon, the "Internet From Sky" proposed by Facebook, and Unmanned Aerial Vehicles (UAVs) to provide temporary broadband coverage in densely populated places such as stadium are some examples of HAP-based communications [5]. Although terrestrial and satellite communication technologies are advancing rapidly in order to make the broadband services accessible to everyone at the cheaper cost, they have their own advantages and disadvantages. In this regard, HAP-based communication is considered as a promising solution which can fulfill the gap between terrestrial and satellite technologies [6], mainly by addressing the coverage limitation in terrestrial communications and the link budget as well as latency issues in satellite communications. In 5G networks, HAP based communication can provide several benefits such as wider coverage areas as compared to terrestrial system, flexible frequency reuse patterns and cell sizes, low cost, incremental and rapid deployment, and easier upgrading 
In time varying wireless environments, accurate knowledge of channel characteristics such as channel impulse response, power profile and delay profile is crucial in order to design efficient transceivers and to provide the desired Quality of Service (QoS) to the end-users. In this regard, various blind [8], semi-blind [9] and training (pilot) sequence based [10] techniques have been proposed in the literature. In the training sequence based techniques, a set of training signal, i.e., pilot signals are transmitted and the characteristics of the channel are estimated based on the received data and the prior knowledge of pilot signals. The main drawback of training pilot sequence based approach is that it requires significant amount of radio resource in terms of dedicated frequency/time slots for the employed pilot sequence. To address this issue, Superimposed Pilot (SiP) sequence based approach, in which a user-specific periodic pilot sequence with low power is superimposed to the corresponding information sequence at the transmitter [11], has recently received important attention $[11,12]$.

In contrast to the conventional terrestrial Rayleigh scattering channel, the wireless channel of an HAP system follows Rician fading distribution due to the presence of strong line of sight component. Authors in [6] have recently evaluated the performance of two widely used channel estimation techniques Least Square (LS) and Linear Minimum Mean Square Error (LMMSE) in term of Mean Square Error (MSE) and Bit Error Rate (BER) versus Signal Noise to Ratio (SNR) considering different elevation angles from HAPs to the user in the ground, velocities of user, and modulation orders. Similarly, authors in [13] evaluated the performance of pilot-based estimation technique for Single Carrier Frequency Division Multiple Access (SC-FDMA) LTE over the HAP scenario. However, the evaluation of superimposed pilot based channel estimation over the HAP channels is missing in the literature.

By exploiting the sparsity of wireless multipath channels, Compressive Sensing (CS) based SiP sequence method has been studied in various contexts such as Single Input Single Output (SISO) systems [14], sparse Multiple Input Multiple Output (MIMO) channels [15] and underwater acoustic channels [16]. However, none of these studies dealt with the design of optimal pilot sequence whose length needs to be adjusted the nature of the propagation channel. In this regard, one of the main contributions of this paper is to apply SiP sequence based channel estimation technique in estimating sparse HAP radio communication channels by using the prior knowledge of users' location at both transmit and receive sides. The remainder of this paper is organized as follows: In Sec. II, the proposed system model along with details of the HAP channel, first order statistics of the received signals, proposed location aware SiP based Stage-wise Orthogonal Match Pursuit (StOMP) algorithm for channel estimation, and implemented equalizer are thoroughly discussed. The simulation setup and numerical results are presented in Sec. III. Finally, the conclusions are discussed in IV.

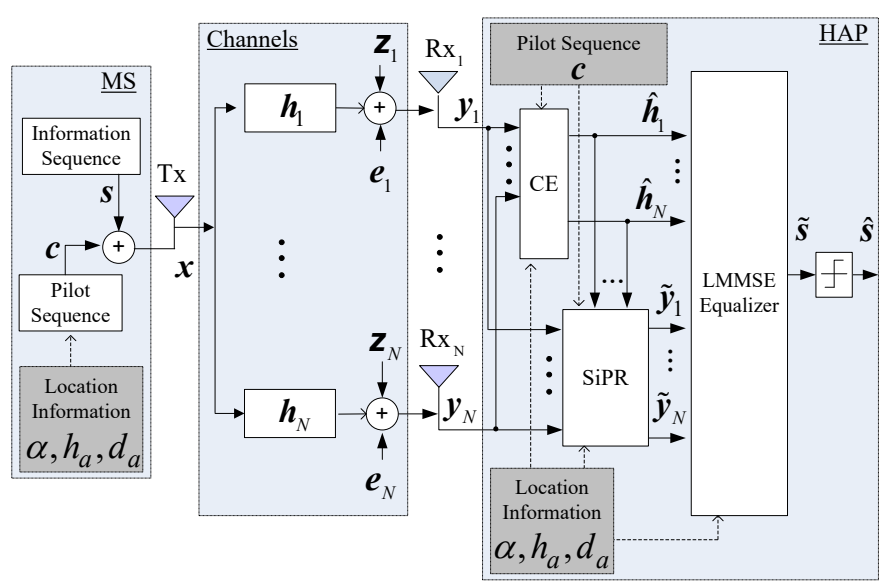

Fig. 1: Block Diagram of proposed system model.

\section{Proposed System Model}

In a typical HAP based land mobile radio cellular network, a high altitude station serves the mobile users at low altitude (at the ground) in a large geographic region; where the backhaul of the network may be ground (preferably) or satellite based. High resolution spatial multiplexing assisted HAP cellular communication systems is considered in this study. In the emerging multiuser wireless communication networks, location of the user(s) is expected to be an agreed public information within the network [?]. In such network, the prior available knowledge of users' location can be exploited in designing pilot sequence and acquiring an accurate channel estimate. Block diagram of the proposed system model for communication of an active Mobile Station (MS) with HAP (i.e., single-input multiple-output (SIMO) communication scenario) with $N$ receive antenna elements is shown in Fig. 1. Let $s=[s(0), s(1), \cdots, s(K-1)]^{H}$ represent the active MS's information sequence. A known deterministic and periodic low-power pilot sequence $\boldsymbol{c}=[c(0), c(1), \cdots, c(K-1)]^{H}$, having period $P$ such that $c(k)=c(k+a P)$ for $k$ and $a$ be any integers, is superimposed (arithmetically added) over the information sequence $s$. The variance of pilot and information sequence is $\sigma_{c}^{2}$ and $\sigma_{s}^{2}$, respectively. The Pilot-to-Information power Ratio (PIR) can thus be defined as, $\psi:=\sigma_{c}^{2} / \sigma_{s}^{2}$. The transmitted sequence can be expressed as, $\boldsymbol{x}=\boldsymbol{s}+\boldsymbol{c}$.

\section{A. Channel(s) Model and Characteristics}

Physical description of the channel between an active MS at ground and HAP is presented in this section. The considered HAP land mobile radio cellular propagation environment is illustrated in Fig. 2. The location of ground MS w.r.t. the elevated HAP can be represented in cartesian coordinates as $\left(d_{a}, 0, h_{a}\right)$; where $d_{a}$ and $h_{a}$ are the horizontal separation of HAP from MS and height of HAP, respectively. With the purpose to archive geometric simplicity, the $\mathrm{X}$-axis is assumed always aligned along the direction of desired user with origin $(0,0,0)$ of the coordinate system taken at base of the HAP. The translation in the origin of coordinates from the base of HAP to the position of MS can be obtained as, $x_{m}=x-d_{a}$, 


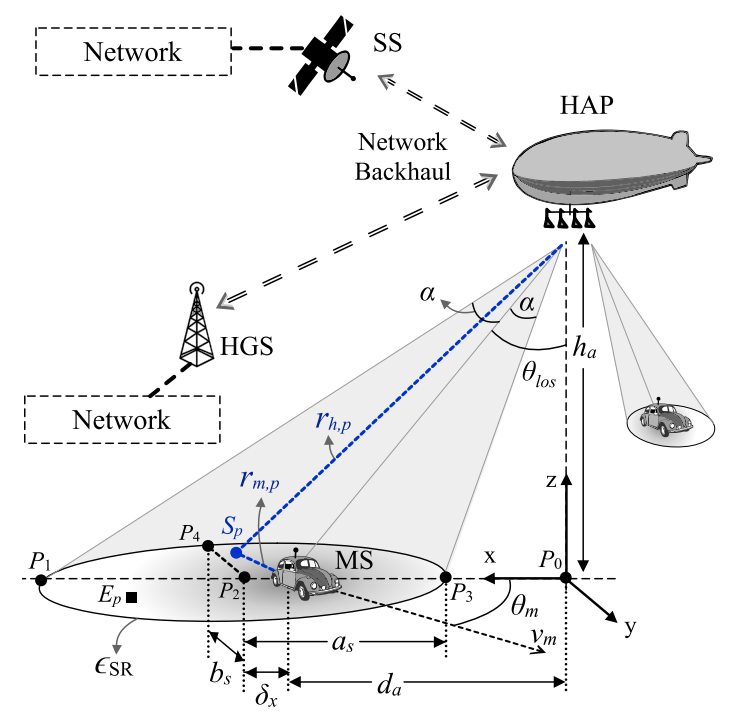

Fig. 2: Geometric composition of emerging HAP based land mobile radio cellular network.

$y_{m}=y$, and $z_{m}=z-h_{a}$. The HAP station is assumed equipped with a flat-top directional antenna with its beamwidth represented by $\alpha$. The boundary of antenna radiation pattern in angular domain is represented by a circular cone pointing directly towards the desired user, i.e., the beam is centered about the ground MS is located at the tip of (center of cone's base-circle) radiation region. The equation for 3D circular cone representing the boundary of the directional antenna's radiation in the cartesian coordinate system can be expressed as,

$\left(h_{a}-z\right)^{2}+x^{2}+y^{2}=\sec ^{2} \alpha\left(x \sin \theta_{l o s}+\left(h_{a}-z\right) \cos \theta_{l o s}\right)^{2}$,

where $\theta_{\text {los }}=\arctan \left(h_{a} / d\right)$ represents elevation LoS angle. Equation of the Scattering Region's (SR) bounding ellipse $\left(\epsilon_{\mathrm{SR}}\right)$ in the vicinity of ground MS, formed by the intersection of the beam (3D cone) with ground plane, can be obtained by substituting $z=0$ in (1). The major and minor dimensions of the elliptical shaped boundary of the SR $\left(\epsilon_{\mathrm{SR}}\right)$ can be expressed as a function of the HAP's antenna beam-width and MS's location information, as given below,

$$
a_{s}=\frac{h_{a} \sin (2 \alpha)}{\cos (2 \alpha)+\cos \left(2 \theta_{l o s}\right)}, \quad b_{s}=\sqrt{\frac{2 h_{a}^{2} \sin ^{2} \alpha}{\cos (2 \alpha)+\cos \left(2 \theta_{l o s}\right)}} .
$$

The area of illuminated SR $\left(\epsilon_{\mathrm{SR}}\right)$ can be obtained as, $A_{s}=\pi a_{s} b_{s}$. The effective scattering objects $S_{p}$ and set of interfering transmitters $E_{p}$ within this illuminated SR $\epsilon_{\mathrm{SR}}$ has a density $\rho_{s}$ and $\rho_{e}$, respectively. The geometric composition of the proposed propagation environment induces a shift in the horizontal position of MS from the center of SR. This shift can be expressed as,

$$
\delta_{x}=\frac{h_{a} \sin \left(2 \theta_{l o s}\right)}{\cos (2 \alpha)+\cos \left(2 \theta_{l o s}\right)}-d .
$$

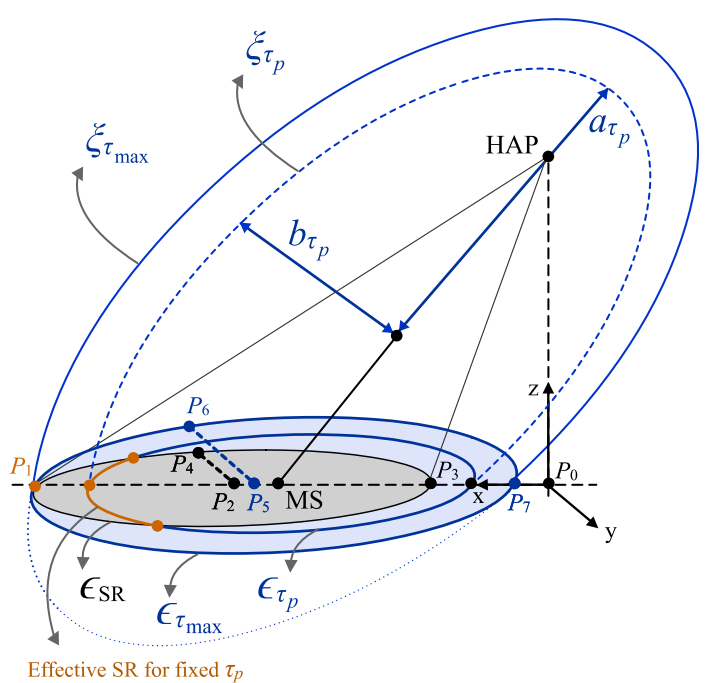

Fig. 3: Effective SR w.r.t. a given path delay $\tau_{p}$.

The shift $\delta_{x}$ will be equal to 0 when the MS is exactly under the HAP, i.e., a circular SR with MS at its center. Length of a particular multipath corresponded from a certain scattering point $\left(S_{p}\right)$ can be obtained as $l_{p}=r_{m, p}+r_{h, p}$; where $r_{m, p}$ and $r_{h, p}$ represent the length of direct distance of $S_{p}$ from MS and HAP, respectively. The spatial scatter density function can be transformed to angular and radial components, by using the following transformation,

$$
p\left(r_{m, p}, \phi_{m}\right)=\frac{f_{x_{m}, y_{m}}^{(\cdot)}\left(x_{m}, y_{m}\right)}{\left|J\left(x_{m}, y_{m}\right)\right|} \mid \begin{aligned}
& x_{m}=r_{m, p} \cos \phi_{m} \\
& y_{m}=r_{m, p} \sin \phi_{m}
\end{aligned}
$$

where $f_{x_{m}, y_{m}}^{(\cdot)}\left(x_{m}, y_{m}\right)$ represents the scatter density function, which may follow a certain distribution in a certain propagation environment. For the cases of uniform scatter density, it can be expressed as, $f_{x_{m}, y_{m}}^{U}\left(x_{m}, y_{m}\right)=1 / A_{s}$ for $\left(x_{m}, y_{m}\right) \in$ $\epsilon_{S R}$. The MS antenna is assumed to have unity gain in all the directions, i.e., isotropic antenna. Incorporating realistic antenna gain patterns is straight forward, the far-end (i.e., HAP in this case) antenna pattern can be included in the definition of scatter density function $f\left(x_{m}, y_{m}, z_{m}\right)$ and observing-end (i.e., ground MS in this case) antenna pattern can be included by directly multiplying it with the plain PDF of AoA $p\left(\phi_{m}\right)$. The delay associated with a multipath corresponded from a certain scattering point $S_{p}$ can be expressed as,

$$
\tau_{p}=\frac{1}{v}\left(r_{m, p}+\sqrt{d^{2}+h_{a}^{2}+r_{m, p}^{2}-2 r_{m, p} d \cos \phi_{m}}\right) .
$$

where $v$ represents the velocity of prorogation of electromagnetic waves. The joint density function given in (5) can be transformed to obtained joint angle and delay density function by using the relationship given in (6), which can expressed as, 


$$
p\left(\tau_{p}, \phi_{m}\right)=\left.\frac{p\left(r_{m, p}, \phi_{m}\right)}{\left|J\left(r_{m, p}, \phi_{m}\right)\right|}\right|_{r_{m, p}=\left(d^{2}-v^{2} \tau_{p}^{2}\right) /\left(2 d \cos \phi_{m}-2 v \tau_{p}\right)}
$$

The closed-form joint AoA and ToA density function can thus be expressed as,

$$
\begin{aligned}
& p\left(\tau_{p}, \phi_{m}\right)= \\
& \frac{\left(d^{2}-v^{2} \tau_{p}^{2}\right)\left(v^{3} \tau_{p}^{2}+v d^{2}-2 d \tau_{p} v^{2} \cos \phi_{m}\right) f_{x_{m}, y_{m}}^{(\cdot)}\left(\dot{x}_{m}, \dot{y}_{m}\right)}{4\left(d \cos \phi_{m}-v \tau_{p}\right)^{3}}
\end{aligned}
$$

where $x_{m}=\left(\left(d^{2}-v^{2} \tau_{p}^{2}\right) \cos \phi_{m}\right) /\left(2 d \cos \phi_{m}-2 v \tau_{p}\right)$ and $\dot{y}_{m}=\left(\left(d^{2}-v^{2} \tau_{p}^{2}\right) \sin \phi_{m}\right) /\left(2 d \cos \phi_{m}-2 v \tau_{p}\right)$. The LoS multipath component correspond in shortest delay, which can be computed as $\tau_{o}=\sqrt{d^{2}+h_{a}^{2}} / v$. The overall longest multipath delay in the proposed scenario is corresponded from the farthest scattering point (i.e., the scattering object at point $\left.P_{1}\right)$, which can be computed as $\tau_{\max }=\left(h_{a}\left(\tan \left(\alpha+\theta_{\text {los }}\right)+\sec \left(\alpha+\theta_{\text {los }}\right)\right)-d\right) / v$. Exploiting the geometric property of an ellipsoid, the sum of distances from foci points of an ellipse to any point on its surface is always the same, the boundary of SR can be drawn with respect to $\tau_{\text {max }}$, as illustrated in Fig. 3. Similarly, for any given delay $\tau_{p}$, an ellipsoid $\left(\xi_{\tau_{p}}\right)$ representing the possible location of a corresponding scattering point can be drawn with its major, intermediate, and minor axes computed as $a_{\tau_{p}}=v \tau_{p} / 2$ and $b_{\tau_{p}}=c_{\tau_{p}}=\sqrt{a_{\tau_{p}}^{2}-d_{\mathrm{los}}^{2} / 4}$, respectively. The effective $\mathrm{SR}$ for a particular given delay is the intersection of this $3 \mathrm{D}$ ellipsoid $\left(\xi_{\tau_{p}}\right)$ and the horizontal elliptical $\left(\epsilon_{S R}\right)$ boundary of SR drawn by the beam of HAP directional antenna in the vicinity of ground MS. The upper limit of delay $\tau_{p}$ is thus dependant on azimuth AoA $\phi_{m}$, which can be computed as,

$$
\tau_{\lim }=\frac{1}{v}\left(r_{\max }+\sqrt{d^{2}-2 d r_{\max } \cos \phi_{m}+h_{a}^{2}+r_{\max }^{2}}\right),
$$

where $r_{\max }$ represents the distance of MS from farthest scattering point in a particular direction $\left(\phi_{m}\right)$, which can be derived as,

$$
\begin{gathered}
r_{\max }=\frac{1}{a_{s}^{2}+b_{s}^{2}+\left(b_{s}^{2}-a_{s}^{2}\right) \cos \left(2 \phi_{m}\right)} \times\left\{-2 b_{s}^{2} \delta_{x} \cos \phi_{m}\right. \\
\left.+\sqrt{2 a_{s}^{2} b_{s}^{2}\left(\cos \left(2 \phi_{m}\right)\left(-a_{s}^{2}+b_{s}^{2}+\delta_{x}^{2}\right)+a_{s}^{2}+b_{s}^{2}-\delta_{x}^{2}\right)}\right\} .
\end{gathered}
$$

Similarly, the joint delay and angle characteristics observed at HAP can also be derived; by replacing the limits of delay and angular parameters with appropriate limits observed on HAP side of the link. The delay span $\tau_{\text {span }}=\tau_{\max }-\tau_{o}$ suggests the amount of resolvable delay line channel taps as, $L=F_{s} \tau_{\text {span }}$, where $F_{s}$ is the sampling frequency. The spatial location of the scattering objects leading to the arrival of multipath components in different channel taps are illustrated in Fig. 3(b), where $\ell$ is the delay line tap index. In a spatial multiplexing communication scenario, when the HAP antenna is equipped with multiple antennas, the wireless propagation channel of an active MS to $n^{\text {th }}$ antenna element of HAP (i.e., serving BS) can be expressed as [17],

$$
h_{n}(\tau, \phi, \theta)=\Xi_{\mathrm{los}}+\sum_{\ell=1}^{L-1} a_{n, \ell} \delta\left(\tau-\tau_{n, \ell}\right) \delta\left(\phi-\phi_{n, \ell}\right),
$$

where $\tau_{n, \ell}$ and $\phi_{n, \ell}$ is delay time and AoA of $\ell^{\text {th }}$ tap, respectively. The LoS path's contribution is, $\Xi_{\text {los }}=$ $a_{n, 0} \delta\left(\tau-\tau_{o}\right) \delta(\phi-0) \delta\left(\theta-\theta_{\text {los }}\right)$. The set of location information can be calculated by incorporating the adjustment in a certain multipath's length in account of antenna elements separations (i.e., $d_{\lambda}=\lambda_{c} / 2 \sim \lambda_{c} / 4 \mathrm{~m}$ ), where $\lambda_{c}=c / f_{c}$. The time-invariant complex amplitude of $\ell^{\text {th }}$ channel tap is represented by $a_{n, \ell}(t)$.

In air-to-ground and ground-to-air propagation environments, typically only a few sparsely located dominant scattering objects correspond in arrival of signal at the receiver, the channel in delay and angular domains is thus sparse [18, 19]. In such scenarios, the time-invariant channel impulse response vector w.r.t. an active mobile user at ground to HAP receiver contains $Q$ non-zero values at delay tap indexes $\breve{\boldsymbol{p}}=$ $\left[\breve{p}_{0}, \breve{p}_{1}, \cdots, \breve{p}_{Q-1}\right]$; where $Q \propto \rho_{s}$. The non-zero tap positions are independent of antenna element index, since the separation between antenna elements is less compared to the distance of HAP from effective scattering points. The time-invariant channel impulse response vector, $\boldsymbol{h}_{n}=\left[h_{n, 0}, h_{n, 1}, \cdots, h_{n, L-1}\right]^{H}$, with $Q$ non-zero eateries can thus be expressed as,

$$
\boldsymbol{h}_{n}= \begin{cases}\neq 0 & ; \ell \in \breve{\boldsymbol{p}} \\ =0 & ; \text { otherwise. }\end{cases}
$$

The impulse response vector of a channel is said to be $Q / L$ sparse, if $\left\{Q=\left\|\boldsymbol{h}_{n}\right\|_{\ell_{0}}\right\} \ll L$.

\section{B. HAP Receiver}

The corresponding received sequence at $n^{\text {th }}$ HAP antenna element at $k^{\text {th }}$ time instant is given by,

$$
y_{n}(k)=\sum_{\ell=0}^{L-1} h_{n, \ell} x(k-\ell)+z_{n}(k)+e_{n}(k),
$$

where $z_{n}(k)$ is Gaussian noise sample at $n^{\text {th }}$ antenna element and $k^{\text {th }}$ time instant, such that $z_{n}(k) \sim \mathcal{N}\left(\mu_{z}, \sigma_{z}^{2}\right)$. The noise samples are iid and uncorrelated with the information sequence. The sum of interference signals caused by the set of interfering MSs illuminated by the beam of HAP directional antenna is represented by $e_{n}(k)$. The power of interference signals from a certain interferer depends upon the spatial location of interferer, antenna gain of HAP in that particular direction, and the channel between interferer and HAP. Assuming spatial distribution of interferers as uniformly distributed within the defined horizontal illuminated region $\epsilon_{S R}$ and the radiation of the antenna beam pattern as flattop within its beam-width, a relationship between power of 
interfering signal and beam-width of HAP antenna can be drawn as, $\sigma_{e}^{2}=\kappa_{e} \rho_{e} A_{s}$, where $\kappa_{e}$ is path-loss dependant constant of proportionality. The composite power of noise and interference signal can be notated as, $\sigma_{z+e}^{2}=\sigma_{z}^{2}+\sigma_{e}^{2}$.

1) First-Order Statistics: Taking expected value of the received signal $y_{n}(k)$ at $n^{\text {th }}$ receiver, it gives

$$
\mathrm{E}\left\{y_{n}(k)\right\}=\sum_{\ell=0}^{L-1} h_{n, \ell} c(k-\ell)+\mu_{n}^{z+e},
$$

where $\mu_{n}^{z+e}$ represents the non-zero mean of the composite noise and interference signal. As the $\mathrm{SiP}$ sequence $c(k)$ is periodic with cyclic frequency $\beta:=2 \pi m / P$, therefore, the $k^{t h}$ sample of pilot sequence $c(k)$ holds the following relationship,

$$
c(k)=\sum_{m=0}^{P-1} c_{m} e^{j \beta_{m} k} \quad \forall k, \quad c_{m}:=\frac{1}{P} \sum_{k=0}^{P-1} c(k) e^{-j \beta_{m} k} .
$$

The $\mathrm{SiP}$ sequence $\{c(k)\}$ is known at the receiver, therefore, the coefficients $c_{m}$ are also known. Using (15) in (14) gives us, $\mathrm{E}\left\{y_{n}(k)\right\}=\sum_{m=0}^{P-1} d_{m} \mathrm{e}^{j \beta_{m} k}$, where

$$
d_{n, m}:=\sum_{\ell=0}^{L-1} c_{m} h_{n, \ell} \mathrm{e}^{-j \beta_{m} \ell} .
$$

This mean $\mathrm{E}\left\{y_{n}(k)\right\}$, of the received signal $\left\{y_{n}(k)\right\}$, is also periodic with cyclic frequencies $\beta_{m}, 0 \leq m \leq P-1$. A meansquare consistent estimate $\hat{d}_{n, m}$ of $d_{n, m}$ for $\beta_{m} \neq 0$ is given by,

$$
\hat{d}_{n, m}=\frac{1}{M} \sum_{k=1}^{M} y_{n}(k) e^{-j \beta_{m} k}
$$

where $M$ is the length of the transmit symbol sequence. As $M \rightarrow \infty, \hat{d}_{n, m} \rightarrow d_{n, m}$. The relationship for $d_{n, m}$ and $\hat{d}_{n, m}$ given in (16) and (17) can used [11] to express,

$$
\hat{\boldsymbol{d}}_{n}=\mathcal{C} \boldsymbol{h}_{n}
$$

where $\hat{\boldsymbol{d}}_{n}=\left[\hat{d}_{n, 0}, \hat{d}_{n, 1}, \cdots, \hat{d}_{n, P-1}\right]^{H}$ and $\mathcal{C}$ is defined as,

$$
\mathcal{C}=\left[\begin{array}{cccc}
c_{0} & c_{0} & \cdots & c_{0} \\
c_{1} & c_{1} \mathrm{e}^{-j \beta_{1}} & \cdots & c_{1} \mathrm{e}^{-j \beta_{1} L} \\
\vdots & \vdots & \ddots & \vdots \\
c_{P-1} & c_{P-1} \mathrm{e}^{-j \beta_{P-1}} & \cdots & c_{P-1} \mathrm{e}^{-j \beta_{P-1} L}
\end{array}\right]
$$

For $P \geq L$ the channel coefficient vector $\boldsymbol{h}_{n}$ can be uniquely recovered. This condition can be ensured by using the user's location information in designing the SiP base sequence at transmitter and estimating the channel at receiver sides. When the composite noise and interference signal is non-zero mean, i.e., $\mu_{n}^{z+e} \neq 0$, the estimate can be obtained by setting $P \geq L+1$, and by omitting the first row in $\mathcal{C}$ and $\hat{d}_{n, 0}$ from $\hat{\boldsymbol{d}}_{n}$.

2) Channel Estimator (CE): This section presents details of the CE block. The CE is implemented using the proposed SiPStOMP method and a notable SiP-LS method in the literature.
SiP-LS based CE: The least squares estimate of $\boldsymbol{h}_{n}$ can be obtained from (18), proposed in [20], as given below, $\hat{\boldsymbol{h}}_{n}^{\mathrm{LS}}=$ $\left(\mathcal{C}^{H} \mathcal{C}\right)^{-1} \mathcal{C}^{H} \hat{\boldsymbol{d}}_{n}$

SiP-StOMP based CE: In order to reconstruct the sparse channel vector $\boldsymbol{h}_{n}$, we propose a location information aided StOMP algorithm [21] for the model presented in (18). It has been established that for large scale sparse recovery problems, StOMP performs much faster than its counterparts such as Orthogonal Matching Pusruit (OMP) and Basis Pursuit (BP). The proposed location information aided StOMP algorithm for the estimation of $n^{\text {th }}$ channel vector is outlined as below,

Input: Matrix $\mathcal{C}$, vector $\hat{\boldsymbol{d}}_{n}$, and threshold $\Upsilon_{i}$.

Output: Channel estimate vector $\hat{\boldsymbol{h}}_{n}^{\text {StOMP }}$.

1) Initialize residual $\boldsymbol{r}_{0}=\hat{\boldsymbol{d}}_{n}$, index set $\Phi_{0}=\emptyset$, and iteration counter $i=1$.

2) Create a set $\Omega_{i}$ consisting of the indices of all elements in the vector, $\Psi_{i}=\mathcal{C}^{H} \boldsymbol{r}_{i-1}$, which are above the threshold $\Upsilon_{i}$.

$$
\boldsymbol{\Omega}_{i}=\left\{j: \boldsymbol{\Psi}_{i}(j) \geq \Upsilon_{i}\right\}
$$

3) Update the index set by $\Phi_{i}=\Phi_{i-1} \cup \Omega_{i}$ and residual by,

$$
\begin{gathered}
\tilde{\boldsymbol{h}}_{n}=\arg \min _{\breve{\boldsymbol{h}}_{n} \in \mathbb{R}^{\Phi_{i}}}\left\|\hat{\boldsymbol{d}}_{n}-\mathcal{C} \breve{\boldsymbol{h}}_{n}\right\|^{2}, \\
\boldsymbol{r}_{i}=\hat{\boldsymbol{d}}_{n}-\mathcal{C} \tilde{\boldsymbol{h}}_{n} .
\end{gathered}
$$

4) Check stopping criteria; if it is not met update index $i=i+1$ and goto step 2 ; if stoping criteria is met, set the final output vector as $\hat{\boldsymbol{h}}_{n}^{\text {StOMP }}=\tilde{\boldsymbol{h}}_{n}$.

The stopping criteria in this case is a fixed amount of maximum iterations, $\Lambda$. In order to avoid false alarms and missed detection, as proposed in [21], the threshold may be set as $\Upsilon_{i}=t_{i}\left\|\boldsymbol{r}_{i}\right\|_{2} / \sqrt{P}$, where $2 \leq t_{i} \leq 3$. When the channel is sufficiently sparse, after the algorithm exits, $\Phi_{i}$ is expected to have no more than $P$ entries and all the non-zeros in $\hat{\boldsymbol{h}}_{n}^{\text {StOMP }}$ are selected in $\Phi_{i}$.

\section{SiP Remover (SiPR)}

The effect of $\mathrm{SiP}$ sequence from the received signal is removed after estimating CIR such that a regularized version of received signal is fed as input to the equalizer, which can be obtained as,

$$
\tilde{y}_{n}(k)=y_{n}(k)-\hat{\mu}_{n}^{z+e}-\sum_{\ell=0}^{L-1} \hat{h}_{n, \ell} x(k-\ell),
$$

where the estimate of the channel can be obtained by using the techniques discussed in the previous sections, i.e., $\hat{\mathbf{h}}_{n}=\hat{\mathbf{h}}_{n}^{L S}$ for the conventional SiP-LS or $\hat{\mathbf{h}}_{n}=\hat{\mathbf{h}}_{n}^{\text {StOMP }}$ for the proposed location information aided SiP-StOMP. The non-zero mean of composite noise and interference signal can be estimated as, $\hat{\mu}_{n}^{z+e}=(1 / K) \sum_{k=1}^{K}\left(y_{n}(k)-\sum_{\ell=0}^{L-1} \hat{h}_{n, \ell} c(k-\ell)\right)$.

\section{LMMSE Equalizer}

After the effect of pilot sequence is removed, the signal is fed to the equalizer's input which estimates the information 


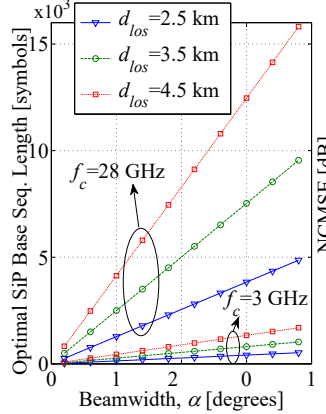

(a)

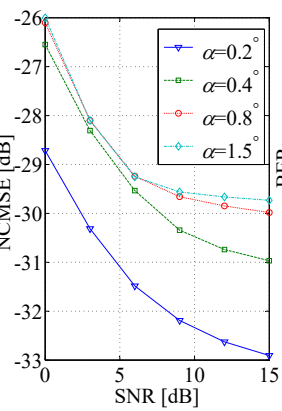

(b)

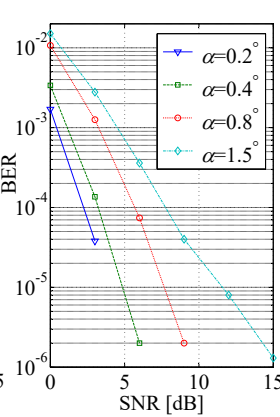

(c)
Fig. 4: a) Optimal length of $\mathrm{SiP}$ base sequence $P$. Effect of beam-width of HAP directional antenna and SNR on NCMSE and BER performance of proposed SiP-StOMP scheme in (b) and (c), respectively. $\left(N=128, K=10^{4}, \Delta=0, d_{l o s}=\right.$ $5 \mathrm{~km}$, and $f_{c}=3 \mathrm{GHz}, \rho_{e}=0.005, \rho_{s}=1, Q / L=1 / 4$, $\Lambda=10$ iterations, and Monte $=100$ runs).

sequence of each user. For, the equalizers part at $n^{\text {th }}$ element of the receiver, the optimal equalizer's weights $\mathbf{w}_{n}$ can be obtained as,

$$
\mathbf{w}_{n}=\left.\left(\hat{\mathbf{H}} \hat{\mathbf{H}}^{*}+2 \hat{\sigma}_{z+e}^{2} \mathbf{I}\right)^{-1} \hat{\mathbf{H}}\right|_{(n-1)\left(L_{e}+L-1\right)+(\Delta+1)^{\prime}},
$$

where $L_{e}$ denotes the length of equalizer, I denotes the $L_{e} \times L_{e}$ identity matrix, $\Delta$ is the decision delay of the equalizer's mappers and $\left.\hat{\mathbf{H}}\right|_{i}$ is the $i^{t h}$ column of $\hat{\mathbf{H}}$. The composite convolutional matrix $\hat{\mathbf{H}}$ of the channel is given as,

$$
\hat{\mathbf{H}}=\left[\begin{array}{llll}
\hat{\mathbf{H}}_{1} & \hat{\mathbf{H}}_{2} & \cdots & \hat{\mathbf{H}}_{N}
\end{array}\right] .
$$

The estimated channels' impulse response vectors can be used to calculate the matrix $\hat{\mathbf{H}}_{n}$, which is shown as below,

$$
\hat{\mathbf{H}}_{n}=\left[\begin{array}{ccccccc}
\hat{h}_{n, 0} & \hat{h}_{n, 1} & \cdots & \hat{h}_{n, L-1} & 0 & \cdots & 0 \\
0 & \hat{h}_{n, 0} & \hat{h}_{n, 1} & \cdots & \hat{h}_{n, L-1} & \ddots & \vdots \\
\vdots & \ddots & \ddots & \ddots & \ddots & \ddots & 0 \\
0 & \ldots & 0 & \hat{h}_{n, 0} & \hat{h}_{n, 1} & \cdots & \hat{h}_{n, L-1}
\end{array}\right] .
$$

Subsequently, the estimate of transmitted information sequence can be obtained as, $\tilde{\mathbf{s}}(k)=$ $\sum_{n=1}^{N} \sum_{\ell_{e}=0}^{L_{e}-1} w_{n, \ell_{e}} \tilde{y}_{n}\left(k-\ell_{e}\right)$. The estimate of user's information sequence $\hat{\mathbf{s}}$, can be obtained after mapping the equalized sequence $\tilde{\mathbf{s}}$ onto a predefined set of symbols. Estimate of the power of composite noise and interference signals can be obtained as,

$$
\hat{\sigma}_{z+e}^{2}=\sum_{n=1}^{N}\left[\left(\frac{1}{K N} \sum_{k=0}^{K} \tilde{y}_{n}^{2}(k)\right)-\sum_{l=0}^{L} \hat{h}_{n, l}^{2}\right]+\kappa_{e} \rho_{e} A_{s} .
$$

\section{RESUlTS AND DisCUSSION}

In this section, a numerical analysis on simulation setup and results is presented. NCMSE and BER are taken as performance metrics. In the simulation setup, the realization of channel between active MS to HAP and interfering MS to HAP are drawn according to the channel description presented in Sec. II-A. Information sequence is drawn from the set of BPSK modulated symbols with an equal probability for both types, i.e., $s \in\left\{+\sigma_{s},-\sigma_{s}\right\}$. A low-power periodic pilot sequence is arithmetically added with the information sequence with its base sequence taken as BPSK modulated zero-mean signal, i.e., $\boldsymbol{c}=\sqrt{\psi \sigma_{s}^{2}} \breve{\boldsymbol{c}}$, where $\breve{\boldsymbol{c}} \in\{+1,-1\}$. The optimal choice of the length of pilot base sequence $P$ is plotted in Fig. 4(a) w.r.t. location of active MS, carrier frequency, and beam-width of HAP's directional antenna. The SNR at $n^{\text {th }}$ receiver is defined as the ratio between variance of received signal and variance of additive noise, i.e., $\mathrm{SNR}_{n}=\sigma_{y_{n}}^{2} / \sigma_{z}^{2}$. The SNR at all the receivers is kept same for all the performed simulations. For each Monte Carlo run, the channel realization, information sequence, $\mathrm{SiP}$ sequence, and noise sequence are generated independently. The effect of beam-width of HAP's directional antenna on NCMSE and BER performance of proposed technique, with amount antennas at HAP taken as $N=128$ and information symbols $K=10^{4}$, is plotted in Fig. 4(b) and (c), respectively. Reducing the beam-width of HAP's directional antenna reduces the area of illuminated spatial region $A_{s}$ in the vicinity of active MS, which further reduces the amount of resolvable multipaths $\left(\tau_{\text {span }}\right)$ and interfering nodes $\left(E_{p}\right)$, therefore an increase in the BER and NCMSE can be witnessed. The plots are obtained for a fixed sparsity level of $Q / L=0.2$. Further, the effect of channels' sparsity level on the performance of proposed SiP-StOMP method is plotted in Fig. 5, along with a comparison with SiP-LS estimation technique proposed in [20]. For the case of non sparse channels, both SiP-StOMP and SiP-LS provide similar results. Whereas, it can be observed that for sparse channels, the proposed SiP-StOMP performs better as compared to SiPLS and the performance gain increases with an increase in channel sparsity. At $\mathrm{SNR}=12 \mathrm{~dB}$, a performance gain of $6.55 \mathrm{~dB}$ and 0.13 is observed in NCMSE and BER performance by the proposed SiP-StOMP compared to SiP-LS. The effect of increasing the PIR (increase in power of SiP sequence compared to information sequences) on NCMSE and BER performance of proposed SiP-StOMP method is plotted in Fig. 6(a) and (b), respectively. Increasing PIR increases the accuracy of channel estimate therefore an increase in NCMSE and BER performance is observed. However, it has a converse behaviour in BER performance for the values of PIR beyond a certain range (i.e., $\psi>0.38$ in this case), which is due to a significant decrease in the power of information sequence. Increasing the amount of HAP antenna elements $N$ (large scale) significantly enhances the BER performance of the system, which can be observed by comparing the results in Fig. R0(c), Fig. R1(b), and Fig. R2(b).

\section{CONCLUSION}

A SiP based method for estimation of sparse multipath channels has been proposed for beamforming assisted HAP land mobile radio communication systems; which exploits the 


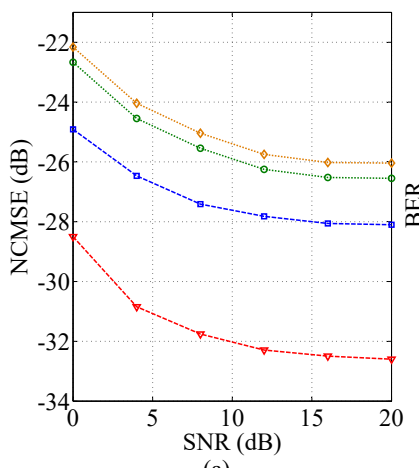

(a)

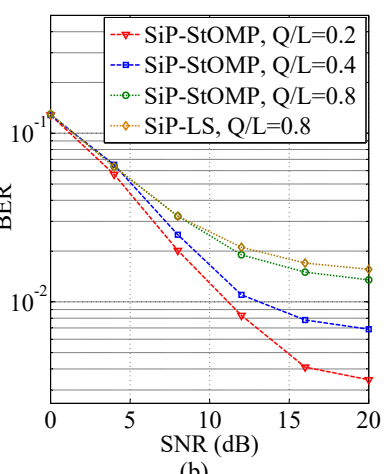

(b)
Fig. 5: Effect of channel sparsity level on the performance of proposed channel estimation method. (a) MSE performance, (b) BER performance, $\left(N=16, K=900, \alpha=0.2^{\circ}, \Delta=\right.$ $0, d_{l o s}=5 \mathrm{~km}, f_{c}=3 \mathrm{GHz}, \rho_{e}=0.02, \rho_{s}=1, \Lambda=10$ iterations, and Monte $=100$ runs.) (a)

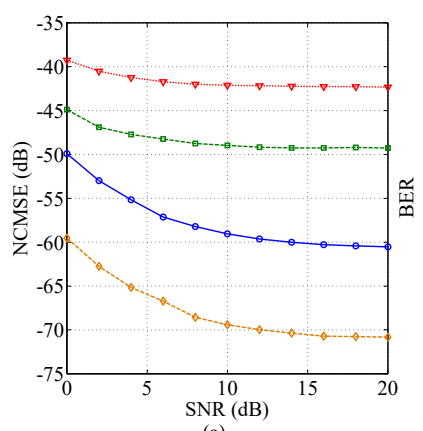

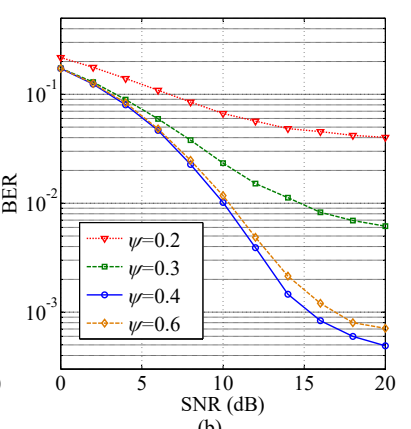

(b)
Fig. 6: Effect of varying PIR on the performance of proposed channel estimation method. (a) MSE performance, (b) BER performance, $\left(N=32, K=2000, \alpha=0.2^{\circ}, \Delta=0, d_{l o s}=\right.$ $8 \mathrm{~km}, f_{c}=3 \mathrm{GHz}, \rho_{e}=0.01, \rho_{s}=1, \Lambda=20$ iterations, and Monte $=100$ runs.)

prior available knowledge of active user's location, spatial density of other interferers, scatter density function, and beamwidth of HAP directional antenna. A comprehensive physical description of the propagation environments between an active mobile user at ground with a multi-antenna HAP has been presented. Mathematical relationship between beam-width of HAP directional antenna, carrier frequency, location of active MS, and delay span of the channel has been proposed, which assists in optimal choice of the length of SiP base sequence. A detailed numerical analysis on NCMSE and BER performance of the system has been conducted, which establishes that the proposed method provides significant performance enhancement for the considered communication scenario when compared to a notable method in the literature. The impact of HAP antenna beam-width, amount of HAP antenna elements, channel's sparsity, SNR, density of interferes, and PIR on the performance of proposed technique has also been thoroughly investigated.

\section{ACKNOWLEDGEMENT}

The first author would like to acknowledge the partial financial support by EU ATOM-690750 research project approved under the call H2020-MSCA-RISE-2015.

\section{REFERENCES}

[1] R. Di Taranto and et al. Location-aware communications for $5 \mathrm{~g}$ networks: How location information can improve scalability, latency, and robustness of 5g. IEEE Signal Processing Magazine, 31(6):102$112,2014$.

[2] A. Dammann and et al. Where2 location aided communications. In 19th European Wireless Conf., pages 1-8, 2013.

[3] S. K. Sharma and et al. Cognitive radio techniques under practical imperfections: A survey. IEEE Commun. Surveys Tutorials, 17(4):18581884, 2015.

[4] S. Yarkan and H. Arslan. Exploiting location awareness toward improved wireless system design in cognitive radio. IEEE Commun. Mag., 46(1):128-136, 2008.

[5] Y. Hu, Y. Hong, and J. Evans. Modelling interference in high altitude platforms with 3D LoS massive MIMO. In IEEE Int. Conf. Commun., pages 1-6, 2016.

[6] M. R. K. Aziz and Iskandar. Channel estimation for lte downlink in high altitude platforms (haps) systems. In Int. Conf. Information and Commun.Technol., pages 182-186, 2013.

[7] T. C. Tozer and D. Grace. High-altitude platforms for wireless communications. Electronics Commun. Engineering J., 13(3):127-137, 2001.

[8] J. K. Tugnait. Blind estimation of digital communication channel impulse response. IEEE Trans. Commun., 42(234):1606-1616, 1994

[9] T. Cui and C. Tellambura. Semiblind channel estimation and data detection for ofdm systems with optimal pilot design. IEEE Trans. Commun., 55(5):1053-1062, 2007.

[10] E. Bjornson and B. Ottersten. A framework for training-based estimation in arbitrarily correlated rician mimo channels with rician disturbance. IEEE Trans. Signal Process., 58(3):1807-1820, 2010.

[11] S. He, J. K. Tugnait, and X. Meng. On superimposed training for MIMO channel estimation and symbol detection. IEEE Trans. on Signal Process., 55(6):3007-3021, 2007.

[12] X. Xie, M. Peng, F. Gao, and W. Wang. Superimposed training based channel estimation for uplink multiple access relay networks. IEEE Trans. on Wireless Commun., 14(8):4439-4453, 2015.

[13] D. Hidayat and Iskandar. Pilot-based estimation for sc-fdma lte in high altitude platforms (haps) channel. In 9th Int. Conf. Telecommun. Systems Services and Applications (TSSA), pages 1-5, 2015.

[14] S. J. Nawaz, K. I. Ahmed, M. N. Patwary, and N. M. Khan. Superimposed training-based compressed sensing of sparse multipath channels. IET Commun., 6(18):3150-3156, 2012.

[15] B. Mansoor, S. J. Nawaz, B. Amin, S. K. Sharma, and M. N. Patwary. Superimposed training based estimation of sparse mimo channels for emerging wireless networks. In 23rd Int. Conf. Telecommun. (ICT), pages $1-6,2016$.

[16] J. Zhao, W. Meng, and S. Jia. Sparse underwater acoustic ofdm channel estimation based on superimposed training. J. of Marine Science and Application, 8(1):65-70, 2009.

[17] A. Klein and W. Mohr. A statistical wideband mobile radio channel model including the directions-of-arrival. In proc. of IEEE Int. Symp. on Spread Spectrum Techniques and Applications, volume 1, pages 102 106, 1996.

[18] R. Sun and D. Matolak. Air-ground channel characterization for unmanned aircraft systems - part ii: Hilly \& mountainous settings. IEEE Trans. on Veh. Technol., PP(99), 2016.

[19] B. Amin, B. Mansoor, S. J. Nawaz, S. K. Sharma, and M. N. Patwary. Compressed sensing of sparse multipath mimo channels with superimposed training sequence. Wireless Personal Communications, In Press, 2016.

[20] J. K. Tugnait and W. Luo. On channel estimation using superimposed training and first-order statistics. IEEE Commun. Letters, 7(9):413-415, 2003.

[21] D. L. Donoho, Y. Tsaig, I. Drori, and J.-L. Starck. Sparse solution of underdetermined systems of linear equations by stagewise orthogonal matching pursuit. IEEE Trans. on Information Theory, 58(2):1094-1121, 2012. 